\title{
The NeuroAiD II (MLC901) in Vascular Cognitive Impairment Study (NEURITES)
}

\author{
Christopher L.H. Chen ${ }^{a}$ Kamran Ikram ${ }^{b}$ Qiu Anqi ${ }^{d}$ Wong Tien Yin ${ }^{b}$ \\ Annabel Chen $^{\mathrm{e}}$ Narayanaswamy Venketasubramanian ${ }^{c}$ \\ for the NEURITE Investigators
}

Departments of a Pharmacology and ${ }^{b}$ Ophthalmology, and ${ }^{c}$ Division of Neurology, National University Health System, and ${ }^{\mathrm{d}}$ Department of Engineering, National University of Singapore, and e Department of Psychology, Nanyang Technological University, Singapore, Singapore

\section{Key Words}

Stroke $\cdot$ Cognitive impairment $\cdot$ Neuroplasticity .

Neuroprotection · Neurogenesis · MLC601 - MLC901 ·

NeuroAiD

\section{Abstract}

Background: A substantial proportion of patients after nondisabling stroke are cognitively impaired compared to age- and education-matched community-dwelling controls. Moreover, poststroke patients who have 'vascular cognitive impairment no dementia' (VCIND) of moderate severity have a high risk of incident dementia, dependency and death. Further studies are urgently needed to demonstrate effective cognition-enhancing therapies in VCIND given the scarcity of evidence-based treatment options. NeuroAiD is a traditional Chinese medicine that has been shown to induce neuroplasticity, promote cell proliferation and stimulate the development of dense axonal and dendritic networks in animal stroke models. NeuroAiD may improve cerebral blood flow and functional recovery after stroke in patients. Objective: To investigate the effects and tolerability of NeuroAiD II in patients with VCIND. Methods: The NeuroAiD II (MLC901) in Vascular Cognitive Impairment Study (NEURITES) is a 24- week, double-blind, randomized, placebo-controlled phase II study of NeuroAiD II in patients with VCIND. The primary outcome is executive function as measured by the Verbal Fluency test. Secondary outcomes include cognitive assessments such as the ADAS-Cog, MoCA, MMSE and Cognitive Battery: activities of daily living as measured by the Alzheimer's Disease Cooperative Study Activities of Daily Living (ADCS-ADL) scale for mild cognitive impairment, behavior as measured by the Neuropsychiatric Inventory, and depression as measured by the Geriatric Depression Scale and the Beck Depression Scale. In addition, there will be novel exploratory outcomes: (a) magnetic resonance imaging of lesion location (structural imaging), structural integrity of white matter pathways (diffusion tensor imaging), neuronal function (resting studies) and perfusion (arterial spin labeling and MR angiography), and (b) retinal and optic nerve imaging. Safety and tolerability will be assessed using adverse events, laboratory tests and vital signs. Conclusions: NEURITES has the potential to set new standards for the systematic evaluation of Asian traditional medicine for integration into standard medicine practice and establishing a novel therapeutic approach for improving cognition after stroke.

Copyright $\odot 2013$ S. Karger AG, Basel

Christopher L.H. Chen

Department of Pharmacology, National University of Singapore, Clinical Research Centre Building MD11, Level 5, 05-09, 10 Medical Drive

Singapore 117597 (Singapore)

E-Mail cplhchen@yahoo.com.sg 


\section{Introduction}

The Importance of Cerebrovascular Disease in

Dementia and Cognitive Impairment

Dementia arising from cerebrovascular disease (CVD), or vascular dementia $(\mathrm{VaD})$, is the second most common form of dementia in the elderly after Alzheimer's disease (AD), constituting 15-20\% of all dementia worldwide [1]. In China and Japan, it may even surpass $\mathrm{AD}$ as the leading cause of dementia, accounting for up to $50 \%$ of all cases [2]. As CVD and stroke increase with ageing and urbanization, the incidence of $\mathrm{VaD}$ is set to rise and emerge as a major public health issue. The public health significance of $\mathrm{VaD}$ is further demonstrated by findings from case-control studies demonstrating that a range of risk factors for $\mathrm{VaD}$ are $\mathrm{CVD}$-related. More importantly, many of these risk factors are modifiable, and efforts aimed at preventing $\mathrm{VaD}$ may be integral in reducing dementia in the elderly.

It is important to note that the conventional definition of $\mathrm{VaD}$ is deficient as stroke may produce a spectrum of cognitive changes but not necessarily prominent memory loss as in AD. The Canadian Study of Health and Ageing prospective cohort study of 10,253 elderly persons reported that 'vascular cognitive impairment' (VCI) was as common as AD. Of subjects with VCI, 'vascular cognitive impairment no dementia' (VCIND) was more common than either $\mathrm{VaD}$ or 'mixed $\mathrm{VaD}$ and AD'. Furthermore, those with VCI had a significantly higher rate of institutionalization and death [3] and half of those who had VCIND developed dementia within 5 years [4]. These findings are similar to previous estimates of the likelihood to progression to dementia in mild cognitive impairment (MCI) individuals [5].

\section{VCIND as Treatment Target}

Previous studies have suggested that a substantial proportion of patients after nondisabling stroke are cognitively impaired compared to age- and education-matched community-dwelling controls [6]. Moreover, those who have VCIND of moderate severity had a higher risk of incident dementia [7] as well as dependency and death [8]. Further studies are urgently needed to better clarify risk factors for deterioration, identify preventive measures to interrupt decline, and demonstrate effective cognition-enhancing therapies.

It is feasible to conduct clinical trials in patients with VCIND and a pilot study has suggested that rivastigmine, an acetylcholinesterase inhibitor, may improve executive function [9], which is in keeping with the neuropsychological profile of cognitive impairment due to CVD [10$11]$.

\section{Nonclinical Studies on NeuroAiD}

Recent pharmacological results show that NeuroAiD (MLC601) and NeuroAiD II (MLC901) improved survival, protected the brain from ischemic injury and decreased functional deficits in animal models of stroke and prevented neuronal death in an in vitro model of excitotoxicity using cultures of cortical neurons exposed to glutamate. In addition, MLC601 and MLC901 have been shown to induce synaptogenesis, promote cell proliferation and stimulate the development of a dense axonal and dendritic network, so as to restore neurological and cellular function in animal models of stroke by processes involved in neurogenesis, neuroplasticity and neuroprotection [12].

Further preclinical evidence for the possible efficacy of NeuroAiD in poststroke cognitive impairment is provided by recent work which has shown MLC901 to prevent necrosis and apoptosis of hippocampal CA1 neurons after global ischemia and improved functional recovery in spatial memory as assessed by the Morris water maze [13].

Hence, NeuroAiD may be effective in improving cognition through these mechanisms in patients with VCIND.

\section{Clinical Studies on NeuroAiD}

Two large double-blind controlled clinical trials were conducted in China, where the efficacy and safety of NeuroAiD was assessed compared to another traditional Chinese medicine (TCM) [14]. In these studies, 605 ischemic cerebral stroke patients (400 in the NeuroAiD group and 205 in the control group) were enrolled in a time window of 2 weeks to 6 months after cerebral infarction. The pooled analysis showed that subjects receiving NeuroAiD were more likely to achieve an independent functional outcome at 1 month than those randomized to the control treatment group (relative risk, 2.4; 95\% CI, 1.28-4.51; $\mathrm{p}<0.007)$. Better improvement in neurological deficit score was in favor of NeuroAiD, although this was not statistically significant (weighted mean difference, 0.22 ; $95 \% \mathrm{CI},-0.11$ to 0.56 ; $\mathrm{p}=0.18$ ). Cognition was not assessed. No severe adverse events were reported and only 2 cases of nausea and vomiting were noted in subjects receiving NeuroAiD.

These results formed the basis of the ongoing CHIMES study in acute stroke [15]. Further safety studies have 
shown that NeuroAiD does not modify hemostasis, hematology, and biochemistry in normal subjects and stroke patients, nor does 3 months of NeuroAiD treatment in acute stroke patients affect laboratory or ECG parameters $[16,17]$.

Recently, it has been shown that NeuroAiD increases cerebral blood flow velocities in stroke subjects, which was associated with improvement in measures of functional recovery [18]. This blood flow increase may be mediated by an effect on stimulating the microcirculation, an important process contributing to neuroplasticity in the central nervous system.

\section{The NeuroAiD II (MLC901) in Vascular Cognitive Impairment Study (NEURITES)}

Traditional medicine is widely used globally in both developing and developed countries and is of rapidly growing health system and economic importance. Although providers of traditional medicine seek better recognition and support, many western-trained professionals have reservations about the benefits and safety of traditional medicine. This conflict between 'uncritical enthusiasm' versus 'uninformed skepticism' can only be resolved by improving the evidence base from which reliable conclusions can be drawn. Not only is there a need for better treatments, the source of which may come from TCM, but it is also vital that efforts are made to identify promising treatments from traditional medicine in a scientifically credible format. Performing well-controlled randomized clinical trials would ensure that potentially beneficial practices are not neglected nor inadequately evaluated practices promoted.

Establishing whether TCM stroke treatment, particularly NeuroAiD with strong clinical and nonclinical basis, can be effective and safe through well-designed clinical trials for VCIND may open up the potential to develop improved treatments based on investigating the active ingredients and mechanisms of actions, as well as address a major public health issue.

NeuroAiD (MLC601) has its origins from TCM and contains 9 herbal and 5 animal components. It is widely available in China and in many countries in Asia. In Europe, a simplified formulation (MLC901) consisting of only the 9 herbal components is available. The simplified formulation of MLC901 was chosen for investigation in this study. NeuroAiD II (MLC901) has been shown to be just as effective as NeuroAiD (MLC601) in preclinical studies of cellular and animal stroke models [12] and to have remarkable neuroprotective and neuroregenerative properties in an animal model of global ischemia [13].
Our hypothesis is that NeuroAiD II is safe and effective in the treatment of poststroke cognitive impairment.

\section{Methods}

\section{Design}

This is a 24-week, double-blind, randomized, placebo-controlled phase II study of NeuroAiD II in patients with VCIND with a planned 1-year extended follow-up period.

\section{Study Population}

Key Inclusion Criteria

Inclusion criteria were the following: male and female patients; age 55-85 years; outpatients; living with a caregiver; Rankin score $\leq 3$; diagnosis of CIND due to CVD; cognitive impairment documented by neuropsychological evaluation within 12 months of index stroke; no history of gradually progressive cognitive deficits before or after index stroke, and not demented by DSM-IV criteria.

\section{Key Exclusion Criteria}

Exclusion criteria were the following: advanced, severe, and unstable disease of any type that may interfere with the efficacy evaluations or put the subject at special risk; no evidence of other neurodegenerative diseases or conditions in addition to CVD that may affect cognition; current diagnosis of dementia or major depression (patients may be included if currently being treated on an antidepressant and stabilized after 3 months); disability that may prevent the subject from completing all study requirements (e.g. blindness, deafness, dysphasia), and intake of any investigational drug in the past 4 weeks or acetylcholinesterase inhibitors or memantine in the past 3 months.

These criteria are in keeping with the recommendations of the American Heart Association/American Stroke Association Statement [19] for Probable Vascular MCI (equivalent to VCIND):

(1) There is cognitive impairment and imaging evidence of CVD and

- there is a clear temporal relationship between a vascular event (e.g. clinical stroke) and onset of cognitive deficits, or

- there is a clear relationship between the severity and pattern of cognitive impairment and the presence of diffuse, subcortical CVD pathology.

(2) There is no history of gradually progressive cognitive deficits before or after the stroke that suggests the presence of a nonvascular neurodegenerative disorder.

(3) The severity of aphasia does not preclude proper cognitive assessment.

(4) There is no evidence of other neurodegenerative diseases or conditions in addition to CVD that may affect cognition such as

- a history of other neurodegenerative disorders (e.g. Parkinson's disease, progressive supranuclear palsy, dementia with Lewy bodies);

- the presence of AD biology as confirmed by biomarkers (e.g. PET, CSF, amyloid ligands) or genetic studies (e.g. PS1 mutation), or

- a history of active cancer or psychiatric or metabolic disorders that may affect cognitive function. 


\section{Randomization}

Study medication will be packed and uniquely identified using a random allocation sequence by staff not involved in the study, using a computer-generated randomization schedule. Randomization will be stratified on 2 major prognostic indicators: (a) Rankin score $=0-1$, Rankin score $=2$, Rankin score $=3$ and (b) subcortical (i.e. lacunar infarct) versus cortical infarct. The randomization list will be kept in a separate location by staff not involved in the trial, who will keep the list private and only reveal treatment allocation upon receiving information demonstrating that the patient is eligible and has consented to the trial.

\section{Study Treatments}

NeuroAiD II consists of a dry extract in capsule form to be taken orally at a dose of 2 capsules 3 times per day. Each $0.4 \mathrm{~g}$ of capsule contains extracts of 9 herbal components (radix astragali, radix salviae miltiorrhizae, radix paeoniae rubra, rhizoma chuanxiong, radix angelicae sinensis, Carthamus tinctorius, Prunus persica, radix polygalae, and rhizoma acori tatarinowii).

The placebo will be produced by the same manufacturer as NeuroAiD II and would contain four constituents sold as food in China and known to have no active effect, namely barley, dried ripe fruit, noodle fish and citric acid, to ensure that the placebo has the same appearance as the active treatment.

\section{Primary and Secondary Outcomes}

To test our hypothesis, NEURITES will primarily evaluate the comparative change from baseline of executive function as measured by the Verbal Fluency test in patients with CIND due to CVD after treatment with either NeuroAiD II or placebo.

Secondary outcome assessments include:

(1) Comparative change from baseline, after treatment with either NeuroAiD II or placebo, of cognitive function, as measured by the ADAS-Cog, MoCA, MMSE and Cognitive Battery (Symbol Digits Modalities test, Maze, Digit Cancellation test, Clock Drawing test, Visual Memory test, Frontal Assessment Battery and Color Trails test 1 and 2).

(2) Effects of NeuroAiD II on activities of daily living as measured on the Alzheimer's Disease Cooperative Study Activities of Daily Living (ADCS-ADL) scale for MCI.

(3) Effects of NeuroAiD II on behavior as measured on the Neuropsychiatric Inventory.

(4) Effects of NeuroAiD II on depression as measured on the Geriatric Depression Scale and the Beck Depression Scale.

(5) Safety assessment using adverse events, laboratory tests and vital signs.

In addition to clinical outcome measures, this study will also incorporate exploratory outcomes which may allow for biomarker discovery and the development of surrogate outcome measures:

(1) Effects of NeuroAiD II on magnetic resonance imaging (MRI) of lesion location (structural imaging), structural integrity of white matter pathways (diffusion-weighted imaging), neuronal function (resting studies) and perfusion (arterial spin labeling and MR angiography).

(2) Effects of NeuroAiD II on retinal and optic nerve imaging.

Novel Exploratory MRI and Retinal Imaging Measures

MRI has been shown to be a predictor of poststroke recovery [20], hence imaging of lesion location (structural imaging), structural integrity of white matter pathways (diffusion tensor imag- ing), neuronal function (resting studies) and perfusion (arterial spin labeling and MR angiography) will be performed at baseline and 52 weeks to analyze treatment effects and relationship with cognitive and functional parameters.

A novel approach is to investigate retinal imaging as the brain shares a similar embryonic origin as the retina. The retinal circulation offers a unique perspective of cerebral microcirculation in vivo and may allow relatively easier access and accurate assessment of the vasculature for studying cerebrovascular pathophysiology and perhaps even the interaction between neurodegenerative pathology and microvasculature. Previous epidemiologic studies showed that signs of retinal vascular changes, as markers of the cerebral microvasculature, are associated with stroke and cognitive impairment. In the Atherosclerosis Risk in Communities study (ARIC) and the ARIC 14-year follow-up study [21], retinal vascular changes were associated with poorer cognitive function and cognitive decline, independent of traditional risk factors. Moreover, in the Cardiovascular Health study, it has been shown that retinal microvascular signs are associated with cognitive impairment assessed by the MMSE, particularly in older hypertensive persons [22]. Advanced computer-based programs will measure novel quantitative retinal vascular parameters which are more indicative of the overall 'optimality state' of the retina and may reflect on the development of diseases from systemic vascular insults (e.g. hypertension, diabetes) in the cerebral microcirculation. We can then attempt to determine cognitive or functional outcomes by retinal vasculature readouts. In addition, since retinal abnormalities have been shown in patients with AD in clinical studies [23], and cognitive functioning is reportedly related to retinal nerve fiber layer thickness measured by scanning laser polarimetry in a large populated-based study [24], optic nerve changes may also be a relevant biomarker in patients with cognitive impairment.

\section{Study Flow}

At baseline, eligible patients will be evaluated for additional inclusion/exclusion criteria, vital signs, neuropsychological tests such as the ADAS-Cog, MoCA, MMSE and Cognitive Battery (Symbol Digits Modalities test, Maze, Digit Cancellation test, Clock Drawing test, Visual Memory test and Frontal Assessment Battery), Color Trails test 1 and 2 and verbal fluency, ADCS-ADL scale for MCI, Neuropsychiatric Inventory, Geriatric Depression Scale, Beck Depression Scale, and past/coexistent medical conditions. MRI and retinal imaging will be performed at baseline and at 52 weeks. Laboratory examinations and ECGs will be evaluated at screening and week 24 . Blood samples for biomarker discovery and measurement of NeuroAiD II components will be taken at baseline and 24 weeks and frozen for future analysis.

At weeks 4 and 8, telephone calls will be made to patients and caregivers to ascertain compliance. At week 12, neuropsychologi$\mathrm{cal}$ and functional measures will be reevaluated. At weeks 16 and 20 , telephone calls will again be made to patients and caregivers to ascertain compliance. At week 24, neuropsychological and functional measures will be reevaluated together with laboratory tests and ECG.

All patients will have their vascular risk factors identified, treated and controlled as per the clinical practice guidelines of the Ministry of Health, Singapore. Information on concomitant medications will be collected as will data on control of vascular risk factors (e.g. blood pressure, glucose and lipids).
Chen et al. 
Fig. 1. Schematic flow of NEURITES. mRS = Modified Rankin Scale; con meds = concomitant medications; $\mathrm{AE}=$ adverse events; $\mathrm{SAE}=$ serious adverse events .

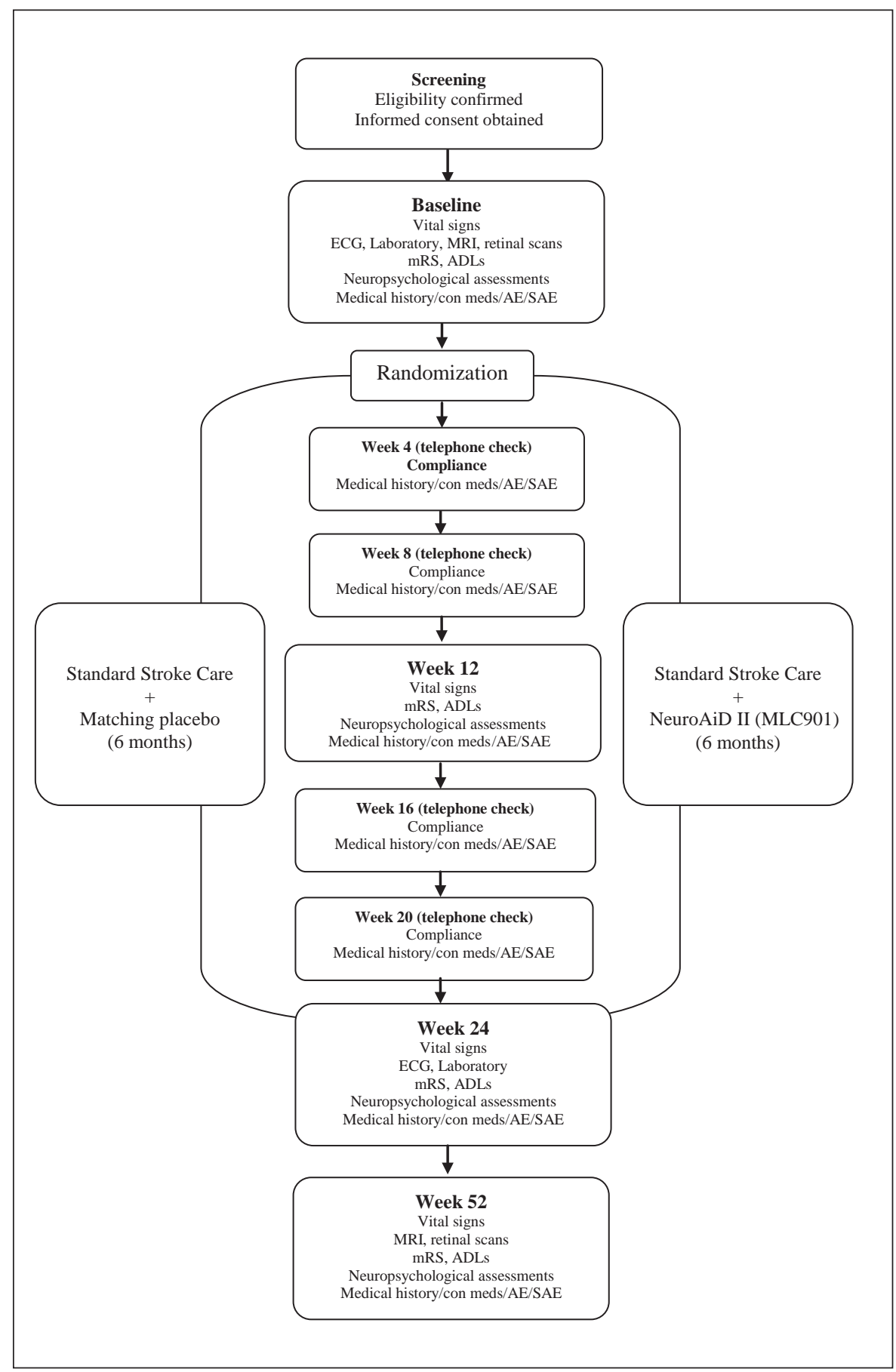

Adverse events and serious adverse events will be captured at every visit. In addition, patients who discontinue the study medication will be followed for safety evaluations through 24 weeks.

At week 52, neuropsychological and functional measures will be reevaluated together with MRI and retinal imaging (fig. 1; table 1).

NeuroAiD in Vascular Cognitive Impairment
Neuropsychological Assessment

A neuropsychological test battery that has been validated for use in Singapore will be administered. The battery assesses six domains, of which four are nonmemory domains. Education-adjusted cutoffs of 1.5 standard deviations below established normal means are used on individual tests. Failure in at least half of the tests in a domain constitutes failure in that domain. 
Table 1. Study visits and assessments in NEURITES

\begin{tabular}{|c|c|c|c|}
\hline \multirow[t]{2}{*}{ Procedure } & \multirow{2}{*}{$\begin{array}{l}\text { Screen- Base- } \\
\text { ing line }\end{array}$} & \multicolumn{2}{|l|}{ Week } \\
\hline & & 48 & $\begin{array}{lllll}12 & 16 & 20 & 24 & 52\end{array}$ \\
\hline
\end{tabular}

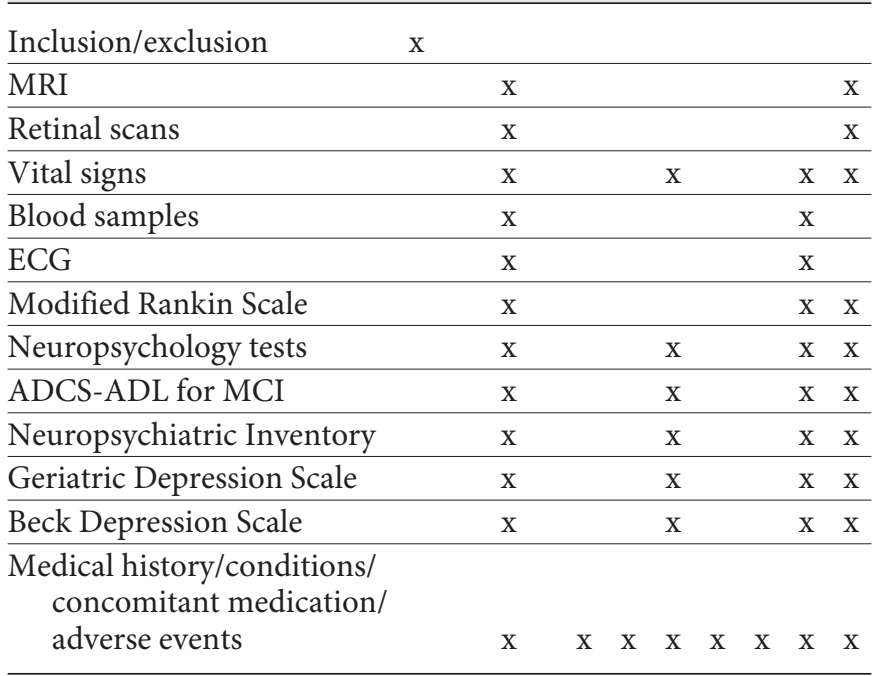

The nonmemory domains include the following: Attention, as defined by digit span, visual span and auditory detection 1; Language, as defined by modified Boston naming and category fluency (animals and food subtasks); Visuomotor speed, as defined by the symbol digit modality test, digit cancellation and maze task, and Visuoconstruction, as defined by Wechsler Memory Scale revised visual reproduction copy task, clock drawing, and Wechsler Adult Intelligence Scale - revised subtest of block design.

The memory domains are: Verbal Memory, as defined by word list recall (immediate, delayed, and delayed recognition) and story recall 1 (immediate and delayed), and Visual Memory, as defined by picture recall (immediate, delayed, and delayed recognition) and Wechsler Memory Scale - revised visual reproduction (immediate, delayed, and delayed recognition).

\section{Determination of VCIND}

As is commonly defined, patients with CIND are impaired in at least one domain of the neuropsychological test battery but do not meet criteria for dementia. In keeping with our previous study, where severity of CIND predicted incident dementia, CIND will be subclassified as CIND-mild (1-2 domains impaired) and CIND-moderate (3-6 domains impaired). VCIND will be determined by information collected at screening. All patients will have either computed tomography or MRI and presence of CVD (i.e. cortical and subcortical infarcts, hemorrhage and white matter disease) documented. Vascular risk factors such as age, diabetes mellitus, hypertension, hyperlipidemia, smoking, ischemic heart disease, peripheral artery disease, as well as history of previous stroke, angina and myocardial infarction, will be ascertained. Stroke subtype will be classified according to the TOAST criteria.

\section{MRI Acquisition}

Every subject will undergo a 1-hour multi-modal MRI scan at baseline and at 52 weeks, including T1-weighted, T2-weighted, FLAIR, diffusion-weighted imaging (HARDI), BOLD and perfusion MRI, and MRA. The MRI scans will be acquired on a 3-tesla whole-body MRI scanner with a state-of-the-art 32-channel head coil. Our imaging protocol starts with a localization scan in three orthogonal planes using fast gradient echo with matrix size of 256 $\times 128, \mathrm{FOV}=240 \mathrm{~mm}$, slice thickness $=5 \mathrm{~mm}$ and number of slices $=3$.

\section{Retinal Imaging}

A Canon CR1 digital nonmydriatic retinal camera will be used to obtain retinal photography after pupil dilation. Retinal photographs will be graded using a semiautomated computer-based program that measures a range of retinal vascular parameters quantitatively, including retinal vascular caliber, vessel tortuosity, branching angles, junctional exponents and fractal dimensions, according to standardized protocols. The grading will be performed by trained retinal photographic graders.

Retina and optic nerve structural imaging will be performed with new spectral-domain optical coherence tomography (SDOCT). High-resolution (up to $3 \mu \mathrm{m})$ and $3 \mathrm{D}$ volume $(200+2 \mathrm{D}$ images) scans will be performed for obtaining cross-sectional images of the retina and optic nerve.

\section{Sample Size}

Data from our pilot study on VCIND showed that the withingroup mean change from baseline in verbal fluency standard deviation was approximately 2.5 , hence the sample size necessary to detect a 2-point difference with a power of $90 \%$ and an alpha of 0.05 is 68 . A target of 100 patients has been set to allow for dropouts. With a standard deviation of approximately $27 \mathrm{~s}$, as determined in the pilot study for the mean change from baseline in the Color Trails 2 test, the difference in population means that can be detected with the same specified power and type I error probability and sample size is $20 \mathrm{~s}$.

\section{Statistical Analyses}

The data will be summarized with respect to demographic and baseline characteristics as well as efficacy and safety observations. Data will be presented for the complete intent-to-treat population (all patients having taken at least 1 dose of study medication) as well as the per-protocol population (all patients who completed the study without major protocol deviations). The safety evaluation analysis compared the means of laboratory tests and vital signs using a 2-sample t test statistic with a 2-tailed alpha of 0.05 . Fisher's exact test was used to compare the proportions of patients who experience an adverse event, a serious adverse event or discontinue medication because of either an adverse or serious adverse event.

The mean change from baseline on the executive function cognitive measures will constitute the primary analysis in this study. A 2-sample t test will be performed with a 2-tailed alpha of 0.05 to test the difference in mean change between placebo and NeuroAiD II groups for the specified primary and secondary outcomes. Where imbalances at baseline are present, linear regression will be used to adjust for baseline scores. All analyses will be performed in an intent-to-treat fashion. 


\section{Conclusions}

There is very promising preclinical and clinical evidence for the role of NeuroAiD in cognitive impairment, particularly for those with underlying vascular etiology. The NEURITE trial has the potential to set new standards for the systematic evaluation of Asian traditional medicine for integration into standard medicine practice and establishing a novel therapeutic approach for improving cognition after stroke. Furthermore, novel MRI and reti- nal imaging measures will be used in assessing treatment effects and relationships with cognitive and functional parameters.

\section{Disclosure Statement}

C.L.H.C. and N.V. have received funding from the National Medical Research Council of Singapore, CHIMES Society and Moleac for NeuroAiD research. K.I., Q.A., W.T.Y. and A.C. have no relevant disclosures.

\section{References}

1 Jorm AF, O’Brien J: The epidemiology of vascular dementia: an overview and commentary; in O'Brien J, Ames D, Gustafson L, et al (eds): Cerebrovascular Disease, Cognitive Impairment and Dementia, ed 2. London, Martin Dunitz, 2004, pp 95-100.

$\checkmark 2$ Chen CP: Transcultural expression of subcortical vascular disease. J Neurol Sci 2004;226: $45-47$.

-3 Rockwood K, Wentzel C, Hachinski V, Hogan DB, MacKnight C, McDowell I: Prevalence and outcomes of vascular cognitive impairment. Vascular Cognitive Impairment Investigators of the Canadian Study of Health and Aging. Neurology 2000;54:447-451.

4 Wentzel C, Rockwood K, MacKnight C, Hachinski V, Hogan DB, Feldman H, Østbye T, Wolfson C, Gauthier S, Verreault R, McDowell I: Progression of impairment in patients with vascular cognitive impairment without dementia. Neurology 2001;57:714716.

5 Ritchie K, Touchon J: Mild cognitive impairment: conceptual basis and current nosological status. Lancet 2000;355:225-228.

6 Tham W, Auchus AP, Thong M, Goh ML, Chang HM, Wong MC, Chen CP: Progression of cognitive impairment after stroke: one year results from a longitudinal study of Singaporean stroke patients. J Neurol Sci 2002; 203-204:49-52.

7 Narasimhalu K, Ang S, De Silva DA, Wong MC, Chang HM, Chia KS, Auchus AP, Chen C: Severity of CIND and MCI predict incidence of dementia in an ischemic stroke cohort. Neurology 2009;73:1866-1872.

8 Narasimhalu K, Ang S, De Silva DA, Wong MC, Chang HM, Chia KS, Auchu AP, Chen C: The prognostic effects of CIND, CIND severity, and domain-specific cognitive impairments in an ischemic stroke cohort. Stroke 2011;42:883-888.

-9 Narasimhalu K, Effendy S, Sim CH, Lee JM, Chen I, Hia SB, Xue HL, Corrales M, Chang HM, Wong MC, Chen CP, Tan EK: Randomized controlled trial of rivastigmine in ischemic stroke patients with CIND. Acta Neurol Scand 2010;121:217-224.
10 Jokinen H, Kalska H, Mäntylä R, Pohjasvaara T, Ylikoski R, Hietanen M, Salonen O, Kaste $\mathrm{M}$, Erkinjuntti T: Cognitive profile of subcortical ischaemic vascular disease. J Neurol Neurosurg Psychiatry 2006;77:28-33.

$\checkmark 11$ Kandiah N, Narasimhalu K, Lee J, Chen CL: Differences exist in the cognitive profile of mild Alzheimer's disease and subcortical ischemic vascular dementia. Dement Geriatr Cogn Disord 2009;27:399-403.

12 Heurteaux C, Gandin C, Borsotto M, et al: Neuroprotective and neuroproliferative activities of NeuroAiD (MLC601, MLC901), a Chinese medicine, in vitro and in vivo. Neuropharmacology 2010;58:987-1001.

13 Quintard H, Borsotto M, Veyssiere J, et al: MLC901, a traditional Chinese medicine, protects the brain against global ischemia. Neuropharmacology 2011;61:622-631.

14 Chen C, Venketasubramanian N, Gan RN, et al: Danqi Piantang Jiaonang (DJ), a traditional Chinese medicine, in poststroke recovery. Stroke 2009;40:859-863.

15 Venketasubramanian N, Chen CL, Gan RN, Chan BP, Chang HM, Tan SB, Picard D, Navarro JC, Baroque AC 2nd, Poungvarin N, Donnan GA, Bousser MG, CHIMES Investigators: A double-blind, placebo-controlled, randomized, multicentre study to investigate Chinese Medicine NeuroAiD Efficacy on Stroke recovery (CHIMES Study). Int J Stroke 2009;4:54-60.

16 Gan R, Lambert C, Lianting J, Chan ESY, Venketasubramanian N, Chen C, et al: Danqi Piantang Jiaonang does not modify hemostasis, hematology, and biochemistry in normal subjects and stroke patients. Cerebrovasc Dis 2008;25:450-456.
17 Young SHY, Zhao Y, Koh A, Singh R, Chan BPL, Chang HM, et al: Safety profile of MLC601 (NeuroAiD) in acute ischemic stroke patients: a Singaporean substudy of the Chinese Medicine NeuroAiD Efficacy on Stroke recovery study. Cerebrovasc Dis 2010; 30:1-6.

18 Bavasard Shahripour R, Shamsaei G, Pakdaman $\mathrm{H}$, et al: The effect of NeuroAiD (MLC601) on cerebral blood flow velocity in subjects' post brain infarct in the middle cerebral artery territory. Eur J Intern Med 2011; 22:509-513.

19 Gorelick PB, Scuteri A, Black SE, Decarli C, Greenberg SM, Iadecola C, Launer LJ, Laurent S, Lopez OL, Nyenhuis D, Petersen RC, Schneider JA, Tzourio C, Arnett DK, Bennett DA, Chui HC, Higashida RT, Lindquist R, Nilsson PM, Roman GC, Sellke FW, Seshadri $S$ : Vascular contributions to cognitive impairment and dementia: a statement for healthcare professionals from the American Heart Association/American Stroke Association. Stroke 2011;42:2672-2713.

20 Stinear C: Prediction of recovery of motor function after stroke. Lancet Neurol 2010;9: 1228-1232.

21 Lesage SR, Mosley TH, Wong TY, Szklo M, Knopman D, Catellier DJ, Cole SR, Klein R, Coresh J, Coker LH, Sharrett AR: Retinal microvascular abnormalities and cognitive decline: the ARIC 14-year follow-up study. Neurology 2009;73:862-868.

22 Baker ML, Marino Larsen EK, Kuller LH, Klein R, Klein BE, Siscovick DS, Bernick C, Manolio TA, Wong TY: Retinal microvascular signs, cognitive function, and dementia in older persons: the Cardiovascular Health Study. Stroke 2007;38:2041-2047.

-23 Guo L, Duggan J, Cordeiro MF: Alzheimer's disease and retinal neurodegeneration. Curr Alzheimer Res 2010;7:3-14.

24 van Koolwijk LM, Despriet DD, Van Duijn CM, Oostra BA, van Swieten JC, de Koning I, Klaver CC, Lemij HG: Association of cognitive functioning with retinal nerve fiber layer thickness. Invest Ophthalmol Vis Sci 2009;50: 4576-4580. 